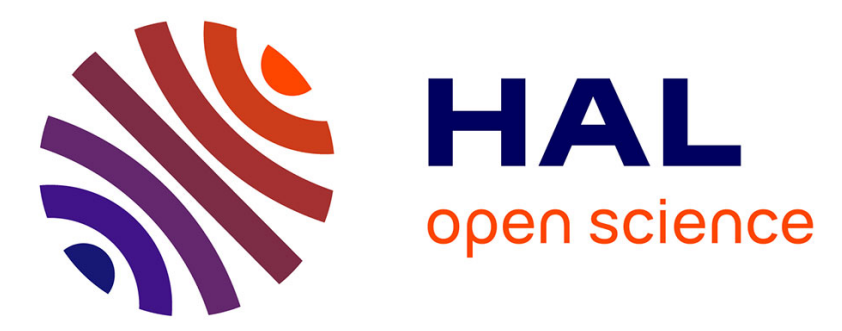

\title{
Road Speed Signatures from C-ITS messages
}

Juliet Chebet Moso, Stephane Cormier, Hacene Fouchal, Cyril de Runz, John

Wandeto, Hasnaa Aniss

\section{To cite this version:}

Juliet Chebet Moso, Stephane Cormier, Hacene Fouchal, Cyril de Runz, John Wandeto, et al.. Road Speed Signatures from C-ITS messages. IEEE International Conference on Communications (ICC 2021), Jun 2021, Montreal, Canada. pp.1-6, 10.1109/ICC42927.2021.9500949 . hal-03319245

\section{HAL Id: hal-03319245 \\ https://hal.science/hal-03319245}

Submitted on 18 Feb 2022

HAL is a multi-disciplinary open access archive for the deposit and dissemination of scientific research documents, whether they are published or not. The documents may come from teaching and research institutions in France or abroad, or from public or private research centers.
L'archive ouverte pluridisciplinaire HAL, est destinée au dépôt et à la diffusion de documents scientifiques de niveau recherche, publiés ou non, émanant des établissements d'enseignement et de recherche français ou étrangers, des laboratoires publics ou privés. 


\title{
Road Speed Signatures from C-ITS messages
}

\author{
Juliet Chebet Moso ${ }^{1,3}$, Stéphane Cormier ${ }^{1}$, Hacène Fouchal ${ }^{1}$, Cyril de Runz ${ }^{2}$, John Wandeto ${ }^{3}$, Hasnaâ Aniss ${ }^{4}$ \\ ${ }^{1}$ CReSTIC EA 3804, 51097, Université de Reims Champagne-Ardenne, France \\ ${ }^{2}$ BDTLN, LIFAT, University of Tours, France \\ ${ }^{3}$ Computer Science, Dedan Kimathi University of Technology, Kenya \\ ${ }^{4}$ COSYS/ERENA, Université Gustave Eiffel, France \\ juliet-chebet.moso@etudiant.univ-reims.fr, \{stephane.cormier, hacene.fouchal $\} @$ univ-reims.fr, \\ cyril.derunz@univ-tours.fr, john.wandeto@dkut.ac.ke, hasnaa.aniss@univ-eiffel.fr
}

\begin{abstract}
Cooperative Intelligent Transport Systems(C-ITS) focus on improving safety, comfort, traffic and energy efficiency. Vehicle speed and other speed based indicators are commonly used parameters in traffic research for generation of driving profiles.The main goal for studying speed variation is to gain a better understanding on why drivers respond in certain ways to $\mathrm{road} /$ traffic conditions and to discover factors which affect their actions. The aim of this paper is to use a real dataset of Cooperative Awareness Messages generated in a C-ITS environment to generate speed signatures. We apply a segmentation technique and statistical analysis in generation and evaluation of road speed signatures. Based on our approach, interesting characteristics on the evolution of driving behavior are revealed from speed signatures.
\end{abstract}

Index Terms-Driver behavior, Speed signatures, incident detection, segmentation.

\section{INTRODUCTION}

The development of Cooperative Intelligent Transport Systems (C-ITS) focuses on improving safety, comfort, traffic and energy efficiency. C-ITS exploits the use of V2X communication where by, data sharing is done using Vehicle-toVehicle communication (V2V) and Vehicle-to-Infrastructure communication (V2I)[1]. C-ITS provide real time information on individual vehicles through the use of Cooperative Awareness Messages (CAM). While Decentralized Environmental Notification Message (DENM) contains information related to an event that has potential impact on road safety or traffic condition (e.g. road works, accidents, vehicle breakdown etc.) resulting in prompt and active incident prevention. However, the impact of C-ITS services on road networks is yet to be fully felt and evaluated due to the novelty of the concept. This can be attributed to the current penetration rate of C-ITS equipped vehicles and their compliance rate[2].

Trajectory data is ordinarily characterized by a raw collection of time ordered spatio-temporal points which capture the motion of an object in geographical space over a time period. Depending on the capability of the device used, these movement track data at every instant of time can include additional attributes like the speed, acceleration, direction of motion etc.The trajectories of moving objects express a concise overview of their behavior.When analyzing the trajectory data, we do not only consider the consolidation of the recorded point data but we are more concerned with extracting and understanding the semantic meaning of the trajectory.

The main goal for studying speed profiles is to gain a better understanding on why drivers respond in certain ways to road/traffic conditions and to discover factors which affect their actions. A key feature is the speed of the vehicle, which has a direct consequence on safety, productivity and the magnitude of environmental impacts for the traffic ecosystem. As an example, very aggressive accelerations result in higher emissions which have a negative impact on the environment.

According to [3] speed can be analyzed in three different ways: "spot measurements (by radar or loop detector), speed profile as a function of time (time-speed profile) or speed profile as a function of space (space-speed profile)". The spacespeed profiles consider speed against the vehicles position (i.e. speed versus distance traveled from an initial point), and is quite useful in driver behavior analysis. Space-speed profiles have been applied in the study of driver behavior in presence of traffic calming measures (speed humps, cushions and chicanes) [4], and at signalized intersections [5].

The information acquired from analysis of vehicle speeds can be useful in identification of black-spots (accident prone locations) and for gaining a better understanding of travel time. Further, it can be used to evaluate the need for infrastructure, for example speed bumps, humps and speed cameras on certain sections of the road. We can also be able to evaluate the impact of the introduction of these infrastructures by analyzing the speed profiles of vehicles as they pass through this sections and comparing it to their behavior before the infrastructure was introduced on the road.

We generate speed signatures from CAMs using segmentation and statistical data analysis approach. We consider the collective movement of vehicles along a particular road segment and evaluate their aggregate driving behavior through analysis of speed signatures.The generated speed signatures from this study will be used for generation of synthetic trajectory data in our subsequent work. We make the following contributions: (a) we present a detailed state of the art on speed profile generation and trajectory segmentation; (b) we propose a segmentation and statistical based methodology for generation and analysis of speed signatures ; (c) we evaluate the methodology using a real dataset of CAM messages 
generated in C-ITS naturalistic driving environment.

The rest of this paper is structured as follows: Section II presents the state of the art investigation on speed profiles generation and analysis techniques. Trajectory segmentation techniques based on speed profiles are also reviewed. Section III presents the problem statement and methodology. Section IV presents the experiments and results, and Section V presents the conclusion and future work.

\section{RELATED WORKS}

This section introduces works on speed profile analysis and trajectory segmentation.

\section{A. Speed profile analysis}

Vehicle speed and other speed based indicators are commonly used parameters in traffic research for generation of driving profiles. Continuous speed data can be analyzed to reveal speed variation patterns over a particular length of road, acceleration behavior and generation of total travel time. Based on speed data collected over a relative length of time and distance, information on individual driver behavior can be extract from analysis of data for a particular vehicle. The research interest may also be on road infrastructure, where the aim is to extract behavior profiles of road users at intersections, roundabouts, speed humps etc. In this kind of scenario, collective analysis of data from all the vehicles passing through these infrastructure sections will provide a better understanding of movement behavior.

In [6], outlier detection and machine learning algorithms were used to detect traffic lights, street crossings and roundabouts by analyzing speed and acceleration signals estimated from GPS data. The proposed techniques gave very good results for street crossings and roundabouts. However, for traffic signal detection, lower precision and recall scores were observed across all classification algorithms when compared to those observed for roundabouts and street crossings. A possible extension to this study for infrastructure detection would be to do collective analysis of the data from all vehicles which traveled along a particular road section.

In [7] vehicle speed profiles are used to detect traffic lights using classification techniques. They test three different ways of deriving features, namely: use of raw speed measurements; uses of image recognition techniques; and use of functional data analysis. A comparative analysis of the derived feature approaches shows that functional description of speed profiles with wavelet transformation outperforms the other two approaches with majority of the tested classifiers. They also show that Random Forests yield an accurate detection of traffic signals, irrespective of the selected feature extraction technique, while keeping a very low confusion rate with stop signs.

Under normal driving, vehicle speed reduces to zero or close to zero as a vehicle approaches a stop sign and then picks up after that. This behavior at stop signs is exploited in [8] where they propose a clustering based stop sign detection method using speed profiles. The deceleration followed by acceleration at stop signs is also used in [9] as a characteristic to detect stop signs from analysis of data generated by on-board car sensors and mobile phone inertial sensors. Extraction of road network properties (intersections and traffic rules) from GPS data was used in [10] to produce high-quality routable maps. Further, it is possible to detect road traffic congestion and incidents in real time using vehicle speed data [11].

C-ITS is currently an active research area with a focus on analysis of communication logs for anomaly detection, communication latency, driver behavior analysis etc. In the study of driver profiles, unsupervised machine learning techniques were applied to a real dataset in [12]. Their results show that driving profiles can be extracted through clustering. An analysis on communication latency was also carried out with results indicating that the achieved latency was within the globally acceptable range for road safety. Vehicle heading angles were also used in [13] to detected driver profiles within the neighborhood of Points of Interest (e.g. traffic lights, yield signs, toll zones etc).

\section{B. Trajectory segmentation}

One of the key trajectory analysis procedures is trajectory segmentation which splits a sequence of data points into a series of sub-sequences comprising homogeneous points based on some defined criteria. Some of the basic criteria can be derived from the attributes of the trajectory. For example, speed can be used to segment a trajectory by setting a threshold for the difference between the minimum and maximum speed within each sub-sequence. We can also set a threshold for the standard deviation of speed within each sub-sequence. Segmentation of trajectories using spatial density and temporal criteria is considered a cluster-based segmentation problem [14]. A common application of cluster-based segmentation is the detection of stop/move patterns [15],[16]. Segmentation methods can be based on time interval, shape of trajectory and semantic meaning of points (stay point based).

According to [17] analysis of traffic anomalies can be carried out by considering the local traffic anomalies or group traffic anomalies. The research which focus on local traffic anomalies partition the road network into independent segments from where they extract individual anomalies of each segment. The key features used are attributes like vehicle speed which are extracted from the independent segments. The extracted features are then applied to detection algorithms (e.g. clustering based, statistical methods, traffic flow models etc.) to extract traffic anomalies. In the group anomaly category, an anomaly in one road segment propagates to other adjacent roads and is analyzed by considering the causal interactions among road segments.

In [18]incident detection is done using a real dataset of GPS data from vehicle traces. The authors divide the road network into segments based on the road type, date, time and prevailing weather conditions. Each road segment is assigned a normal average speed range e.g. $80-120 \mathrm{~km} / \mathrm{h}$ for a motorway type on weekday's peek time. The segments whose average traveling speed is considerably lower than the assigned normal speed 
are considered abnormal and are extracted. The anomalous segments are further divided into smaller segments so as to isolate the potential incident area. A segment with an incident causing a blockage will have the average speed of the segment in front of it being higher with fewer vehicles. They also consider the behavior of individual vehicles from the anomalous road segments where they identify vehicles with significantly lower speed than the sub-segment current average speed, stopped vehicles and those moving in a different direction to the traffic flow. The challenge with this approach is that the segmentation process is affected by the accuracy of polygonal line coordinates. Also the accuracy range of GPS affects the distinction of incidence from normal traffic congestion.

Long-term traffic anomaly detection (LoTAD) is proposed in [19] where the main aim is to identify long term anomalous traffic regions in urban cities. In this approach, the road network is partition into segments based on bus line data and a real bus trajectory dataset is also segmented based on time slots into temporal and spatial segments (TS-segments) which depict city wide traffic situation. Two trajectory features are extracted, average velocity which defines the traffic condition and average stop time which defines the travel demand. A computation of an anomaly index for TS-segments is done to identify anomalous segments in each bus line which are then consolidated to form anomalous regions. The information extracted from the anomalous regions can be used to offer recommendations for future urban traffic planning.

In [20] Filter-Discovery-Match (FDM) technique is proposed which uses speed patterns to identify incident scenes by partitioning a road network into segments. Speed vectors are generated for consecutive sequences of road segments traveled by a vehicle based on average speed. Real incident data is used to identify incident sections of the road from where incident speed vectors are extracted from vehicles which passed through these sections at the time of the incident. Normal speed vectors are extracted from the road segments by averaging the speed of the vehicles which passed the segments at a specific time frame and were not affected by the traffic incident. Candidate speed patterns are extracted by computing the speed difference between incident speed vectors and normal speed vector for each segment. These candidate patterns are then clustered using K-means algorithm. Extensive experiments with real taxi data and simulated data revealed that FDM yielded a lower mean time-to-detect (MTTD) than the compared existing techniques.

\section{Problem Statement and Methodology}

\section{A. Problem Statement}

The vehicles in a C-ITS environment exchange a lot of messages. Every message contains the position and movement information of the vehicle for the case of CAMs and the prevailing road condition and hazard information in the case of DENMs. The high frequency of generation of CAMs makes it possible to extract a concise mobility pattern of a vehicle. However, due to the current penetration rate of C-ITS equipped vehicles [2] there is a challenge of insufficient amount of data for a more detailed analysis of driving behavior on a microscopic level (route level). We consider a situation where we have mobility data captured for a specific road section for a number of months but with a single trajectory per day for one or two vehicles and missing data for some days. Also, the trajectories were collected at different times of the day. With this kind of data, hourly or daily mobility analysis might not give comprehensive results since we have very few trajectories at varying times of the day.

The generation of simulated data is generally used to solve the data insufficiency issue but this still raises another issue, how to generate realistic data such that the mobility patterns are indistinguishable from those of real vehicles. In this paper we analyze CAM messages with a purpose of generating collective road level speed signatures through the application of a segmentation approach and statistical analysis techniques. We consider the collective movement of vehicles along a particular road segment and evaluate their aggregate driving behavior through analysis of speed signatures. The generated speed signatures from this study will be used for generation of synthetic trajectory data in our subsequent work. A possible application area for the generated speed signatures is in Autonomous vehicles where the signatures can be used as inputs in order to check if the driving is following normal speed/ expected naturalistic speed behavior.

\section{B. Methodology}

In a C-ITS environment cooperative awareness is achieved through exchange of CAMs which contain position information. The collection of mobility data is done through On Board Units installed in the vehicles, road side units among other techniques. These data contains details that explain the movement of vehicles. Each trajectory of a vehicle is considered a multi-attribute, time-ordered sequence of locations traversed and is viewed as a trip.

In our study we used a real dataset of Cooperative Awareness Messages (CAM) collected in France between September 2018 and August 2019 under a C-ITS project [21].During this project, C-ITS CAM messages were generated by vehicles under naturalistic driving environment. The purpose of CAMs is to give dynamic information about the vehicle (i.e. speed, position, heading (direction of motion with regard to true north) etc.). A vehicle sends CAMs to its neighborhood using V2V or V2I communications. The frequency of CAM message generation varies from $10 \mathrm{~Hz}$ to $1 \mathrm{~Hz}(100$ milliseconds to 1000 milliseconds). Each message has an identifier (stationid) associated with the transmitting vehicle but this vehicle is unknown. The message also includes a timestamp, latitude, longitude, altitude, speed, heading angle and the drive direction. The variables latitude, longitude, altitude are the three position variables. The speed, heading angle and drive direction are used as variables of the behavior of the transmitting vehicle. For the purpose of this study, we consider 32 trajectories generated by vehicles traveling through route nationale N118. 
Definition: Trajectory: A raw trajectory consists of a sequence of $n$ points $T=\left[p_{1}, p_{2}, \ldots, p_{n}\right]$, in which $p_{i}=$ $x, y, z, t, A$, where $x, y, z$ represent the position of the moving object in space, $t$ is the timestamp and $A$ represents other attributes associated with the point (i.e. speed, heading angle and drive direction)

In this paper a trajectory is considered as the consolidation of messages uniquely identified by a single identifier.

\section{Experimental Evaluation AND REsults}

We performed trajectory mining using PostgreSQL database with the spatial extension PostGIS used for storing and processing spatial data. Statistical data analysis was done using $\mathrm{R}$ software. We focus our analysis on the messages generated by vehicles which passed through route N118. The entry point for the study area is the section connecting N118 from route La Francillienne, while the exit point is the section connecting to Grande Rue as shown in Fig.1. All the trajectories entering N118 through La Francillienne are considered to be moving in upstream direction. The trajectories moving along N118 in the opposite direction, exiting to La Francillienne are considered downstream trajectories. A total of 32 trajectories are considered with 14 upstream and 18 downstream trajectories.

The aim of our study is to generate speed signatures by modeling speed variations from the start point to end point of route N118. In order to ensure that all the vehicles were positioned on the correct road segment, we performed map matching using PostGIS. We started by converting the latitude and longitude values for each message to a geometry data type using Spatial Reference Systems SRID 4326 (WGS 84) which is the projection for Europe. This allows us to view the graphical output of our queries directly in pgAdmin against an OpenStreetMap background map using PostGIS geometry viewer. Route N118 has both an upstream and downstream section, therefore to identify the trajectories on each section we performed map matching for a few points of each trajectory. Based on the direction indications on OSM background map we were able to label each trajectory as either upstream or downstream.

Considering the fact that the messages are highly sampled with a maximum of up to 10 points per second, we opted to follow an aggregated approach for speed signature generation. The next step is to group the messages based on equal length segments from a specific reference point such that each

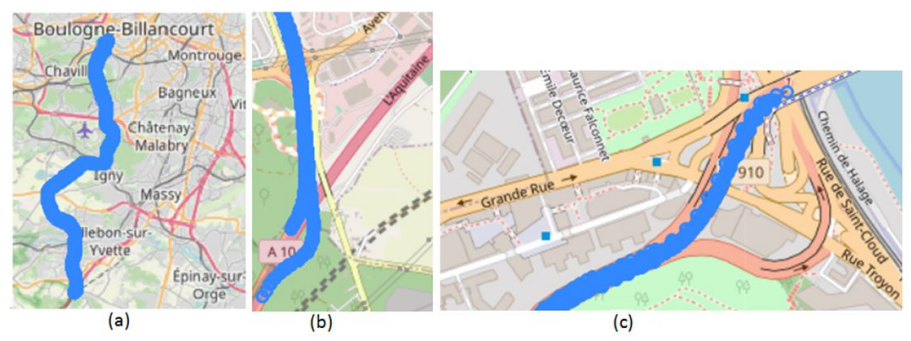

Fig. 1. Area of interest; (a) trajectories on the full stretch of route N118, (b) Entry point, (c) Exit point
TABLE I

SUMMARY OF VEHICLE SPEED

\begin{tabular}{|l|l|l|}
\hline Measure & Upstream & Downstream \\
\hline Minimum & 0.00 & 0.00 \\
\hline 1st Quartile & 16.60 & 20.92 \\
\hline Median & 50.58 & 65.09 \\
\hline Mean & 50.28 & 57.48 \\
\hline 3rd Quartile & 82.33 & 88.63 \\
\hline Maximum & 123.59 & 131.00 \\
\hline Standard deviation & 34.02 & 35.49 \\
\hline
\end{tabular}

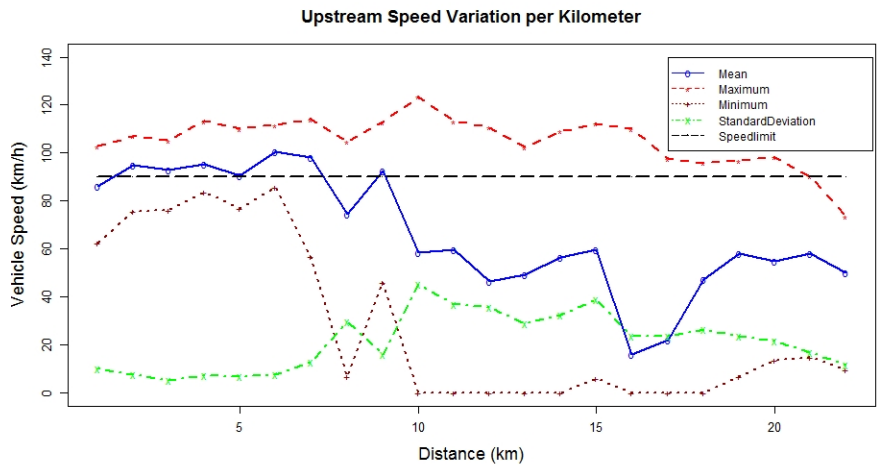

Fig. 2. Upstream Speed signature variation.

message is in a unique segment. We chose the first reference point to be the geometry of the first message at the entry point of route N118. Then using the ST_DWithin function in PostGIS to create a bounding box, we extracted all messages within a 1 kilometer radius of the reference message, this group forms the first segment and is extracted and stored. The second segment is generate from the remaining messages using the same process by moving the reference point to the first message of the unprocessed points at the edge of the previous bounding box. This process iterates until we have all the messages grouped in 1 kilometer length segments. Using the extracted segments we generated aggregate statistics where we computed the minimum, maximum, mean, median and standard deviation for both upstream and downstream sections of the road. Table I presents a summary of vehicle speed in kilometers per hour along route N118.

Based on these aggregates we sort to find answers the following questions:

- Based on the speed signatures, what is the observed evolution of the driving behavior on the road?

- Do drivers maintain the speed limit restriction in force on the road segment?

To answer these questions, we evaluated the variation in the aggregate measures and compared against the speed limits on the road ${ }^{1}, 90 \mathrm{~km} / \mathrm{h}$ on the upstream sections and $80 \mathrm{~km} / \mathrm{h}$ in the downstream section. The results for upstream and downstream speed signatures are shown in Fig. 2 and Fig. 3 respectively. The variation on median speed per segment is shown in Fig.4

\footnotetext{
${ }^{1}$ https://truck-simulator.fandom.com/wiki/N118_(France)
} 


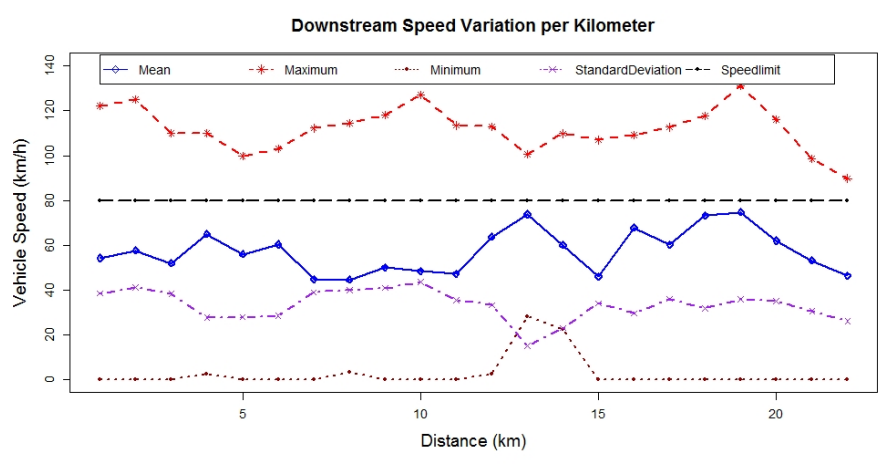

Fig. 3. Downstream speed signature variation.

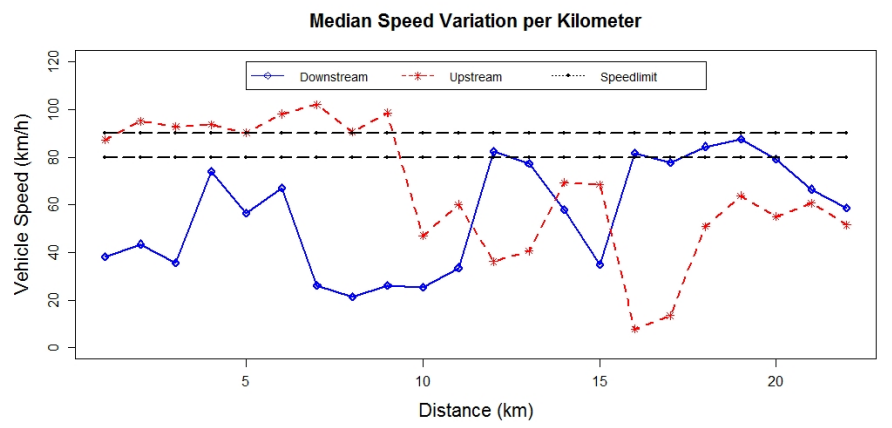

Fig. 4. Median speed signature variation.

Based on our analysis of the upstream section, the average speed varies slightly above the $90 \mathrm{~km} / \mathrm{h}$ limit for the first 8 segments, then there is a decline with the most notable change being at the 11th segment. This behavior is also confirmed by the trend of the standard deviation which increases from the 6th to the 11th segment. This may be attributed to the curvature of the road, an incident or existence of traffic calming infrastructure. On the downstream section, the average speed is more steady and under the speed limit. Based on the analysis of the median speed variation we can conclude that for most parts of the road in both upstream and downstream, the speed limit policy is maintained

It will be of interest to do a more detailed analysis of the data to identify factors causing or influencing the observed behavior, especially on the spike points. Further, useful knowledge can be gained by considering the individual behavior of the vehicles on these particular road segments. This can be done through outlier detection in order to identify incident points or unique behavior among certain drivers. The DENM messages can also be used to extract points where incidents were reported and then use these locations as POIs for traffic incident detection. By extracting the speed signatures of road segments, it could now be possible to discover whether there is a change in driver behaviors over a road segment on a time period (and therefore discover road incidents) by comparing them with the road segment speed signature using anomaly detection method such as Isolation Forest (IForest), Angle-Based
Outlier Detector (ABOD), K-Nearest Neighbours (KNN) etc. [22].

\section{CONCLUSION}

In this work we considered the analysis of speed signatures generated from C-ITS messages with the aim of understanding driving behavior evolution under a naturalistic environment. We have shown that with the application of segmentation and aggregate statistics, one is able to get a better understanding of general driving behavior and also infer information that relates to the road condition and traffic situation. With the current uptake of C-ITS there remains a challenge of insufficient amount of data for a more detailed analysis of driving profiles on a microscopic level (route level). Due to this challenge as future work, we intent to generate realistic synthetic data using the real speed signatures generated from this study.

\section{ACKNOWLEDGMENT}

This work was made possible by EC Grant No. INEA/CEF/TRAN/A2014/1042281 from the INEA Agency for the SCOOP project. Also a PhD scholarship grant from the French Embassy in Kenya. The statements made herein are solely the responsibility of the authors

\section{REFERENCES}

[1] H. Fouchal, E. Bourdy, G. Wilhelm, and M. Ayaida, 'A Framework for Validation of Cooperative Intelligent Transport Systems', in 2016 IEEE Global Communications Conference (GLOBECOM), Washington, DC, USA, Dec. 2016, pp. 1-6, doi: 10.1109/GLOCOM.2016.7841500.

[2] M. Lu, O. Turetken, O. E. Adali, J. Castells, R. Blokpoel, and P. Grefen, 'C-ITS (Cooperative Intelligent Transport Systems) deployment in Europe - challenges and key findings',In 25th ITS World Congress, p. 11, 2018.

[3] C. Andrieu, G. Saint Pierre and X. Bressaud, 'Estimation of space-speed profiles: A functional approach using smoothing splines', In 2013 IEEE Intelligent Vehicles Symposium (IV), pp. 982-987, June 2013.

[4] H. Barbosa, M. Tight and A. May, 'A model of speed profiles for traffic calmed roads', Transportation Research Part A: Policy and Practice, vol. 34, no. 2, pp. 103-123, 2000.

[5] A. Laureshyn, K. Åström and K. Brundell-Freij, 'From speed profile data to analysis of behaviour', IATSS Research, vol. 33, no. 2, pp. 88-98, 2009.

[6] M. Munoz-Organero, R. Ruiz-Blaquez, and L. Sánchez-Fernández, 'Automatic detection of traffic lights, street crossings and urban roundabouts combining outlier detection and deep learning classification techniques based on GPS traces while driving', Computers, Environment and Urban Systems, vol. 68, pp. 1-8, Mar. 2018, doi: 10.1016/j.compenvurbsys.2017.09.005.

[7] Y. Méneroux, A. Le Guilcher, G. Saint Pierre, M. Ghasemi Hamed, S. Mustière, and O. Orfila, 'Traffic signal detection from in-vehicle GPS speed profiles using functional data analysis and machine learning', International Journal of Data Science and Analytics, vol. 10, no. 1, pp. 101-119, 2019, doi: 10.1007/s41060-019-00197-x.

[8] S. Dadras, H. Jamshidi, S. Dadras, and T. E. Pilutti, 'Novel Stop Sign Detection Algorithm Based on Vehicle Speed Profile', in 2019 American Control Conference (ACC), Philadelphia, PA, USA, Jul. 2019, pp. 3994-3999, doi: 10.23919/ACC.2019.8814880.

[9] H. Qiu, J. Chen, S. Jain, Y. Jiang, M. McCartney, G. Kar, F. Bai, D.K Grimm, M. Gruteser, and R. Govindan, 'Towards robust vehicular context sensing', IEEE Trans Veh Technol, vol. 67, no. 3, pp.1909-1922, 2018.

[10] J. Wang, C. Wang, X. Song, and V. Raghavan, 'Automatic intersection and traffic rule detection by mining motor-vehicle GPS trajectories', Computers, Environment and Urban Systems, vol. 64, pp.19-29, 2017. 
[11] E. D'Andrea, and F. Marcelloni, 'Detection of traffic congestion and incidents from GPS trace analysis'. Expert Systems with Applications, vol.73, pp.43-56, 2017.

[12] Leblanc B., Bourdy E., Fouchal H., de Runz C., Ercan S. (2019) Unsupervised Driving Profile Detection Using Cooperative Vehicles' Data. In: Hilt B., Berbineau M., Vinel A., Jonsson M., Pirovano A. (eds) Communication Technologies for Vehicles. Nets4Cars/Nets4Trains/Nets4Aircraft 2019. Lecture Notes in Computer Science, vol 11461. Springer, Cham.

[13] B. Leblanc, H. Fouchal and C. de Runz, 'Driver Profile Detection Using Points of Interest Neighbourhood', 2019 IEEE 90th Vehicular Technology Conference (VTC2019-Fall), Honolulu, HI, USA, 2019, pp. 1-4, doi: 10.1109/VTCFall.2019.8891118.

[14] M. L. Damiani, F. Hachem, Ha. Issa, N. Ranc, P. Moorcroft, and F. Cagnacci, 'Cluster-based trajectory segmentation with local noise', Data Mining and Knowledge Discovery,vol. 32, no. 4 ,2018, pp. 1017-1055.

[15] C. Parent, N. Pelekis, Y. Theodoridis, Z. Yan, S. Spaccapietra, C. Renso, G. Andrienko, N. Andrienko, V. Bogorny, M. L. Damiani, A. Gkoulalas-Divanis, and J. Macedo, 'Semantic trajectories modeling and analysis', ACM Computing Surveys, vol. 45, no. 4, 2013, pp.1-32, doi: $10.1145 / 2501654.2501656$

[16] S. Spaccapietra, C. Parent, M. L. Damiani, J. A. de Macedo, F. Porto, and C. Vangenot, 'A conceptual view on trajectories', Data and Knowledge Engineering, vol. 65, no. 1, 2008, pp. 126-146. doi:10.1016/j.datak.2007.10.008

[17] M. Zhang, T. Li, Y. Yu, Y. Li, P. Hui and Y. Zheng, 'Urban Anomaly Analytics: Description, Detection and Prediction,' in IEEE Transactions on Big Data,2020, doi: 10.1109/TBDATA.2020.2991008.

[18] S. Kamran and O. Haas, 'A Multilevel Traffic Incidents Detection Approach: Identifying Traffic Patterns and Vehicle Behaviours using real-time GPS data,' 2007 IEEE Intelligent Vehicles Symposium, Istanbul, 2007, pp. 912-917, doi: 10.1109/IVS.2007.4290233.

[19] X. Kong, X. Song, F. Xia, H. Guo, J. Wang, and A. Tolba, 'LoTAD: Long-term traffic anomaly detection based on crowdsourced bus trajectory data,' World Wide Web, vol. 21, no. 3, 2018, pp. 825-847, doi: 10.1007/s11280-017-0487-4

[20] X. Han, T. Grubenmann, R. Cheng, S. C. Wong, X. Li and W. Sun, "Traffic Incident Detection: A Trajectory-based Approach," 2020 IEEE 36th International Conference on Data Engineering (ICDE), Dallas, TX, USA, 2020, pp. 1866-1869, doi: 10.1109/ICDE48307.2020.00190.

[21] Scoop.developpement-durable.gouv.fr. 2020. General Presentation SCOOP Project : Connected Road And Vehicle. [online] Available at: ihttp://www.scoop.developpement-durable.gouv.fr/en/generalpresentation-a9.htmli [Accessed 20 September 2020].

[22] Y. Zhao, Z. Nasrullah, and Z. Li, 'PyOD: A Python Toolbox for Scalable Outlier Detection', Journal of machine learning research (JMLR), vol.20, no.96, pp.1-7. 